\title{
1, 25-Dihydroxyvitamin D Measurement
}

National Cancer Institute

\section{Source}

National Cancer Institute. 1, 25-Dihydroxyvitamin D Measurement. NCI Thesaurus. Code C92267.

The determination of the amount of 1, 25-Dihydroxyvitamin D present in a sample. 